\title{
Urinary iodine concentrations of pregnant women in Ukraine
}

Yui Sekitani ${ }^{1}$, Naomi Hayashida ${ }^{1}$, Jumpei Takahashi ${ }^{2}$, Alexander A. Kozlovsky ${ }^{3}$, Stanislav Rudnitskiy ${ }^{4}$, Anjelika Petrova ${ }^{4}$, Oleksandr K. Gutevych ${ }^{4}$, Sergiy A. Chorniy ${ }^{4}$, Shunichi Yamashita ${ }^{5}$ and Noboru Takamura ${ }^{1, *}$

${ }^{1}$ Department of Global Health, Medicine and Welfare, Atomic Bomb Disease Institute, Nagasaki University Graduate School of Biomedical Sciences, Nagasaki, Japan

${ }^{2}$ Center for International Collaborative Research, Nagasaki University, Nagasaki, Japan

${ }^{3}$ Gomel State Medical University, Gomel, the Republic of Belarus

${ }^{4}$ Zhitomir Inter-Area Medical Diagnostic Center, Korosten, Zhitomir, Ukraine

${ }^{5}$ Department of Radiation Medical Sciences, Atomic Bomb Disease Institute, Nagasaki University Graduate School of Biomedical Sciences, Nagasaki, Japan

Running title: Urinary iodine in Ukrainian pregnant women

\section{Correspondence:}

Noboru Takamura, M.D., Ph.D. 
Professor and Chairman

Department of Global Health, Medicine and Welfare, Atomic Bomb Disease Institute,

Nagasaki University Graduate School of Biomedical Sciences

1-12-4 Sakamoto, Nagasaki 8528523, JAPAN

TEL: +81-95-819-7170

FAX: +81-95-819-7172

E-mail: takamura@nagasaki-u.ac.jp

Word count: 2584 words

Tables and Figures: 2 tables and 1 figure

References: 22 


\section{Abstract}

Background: Iodine requirements increase during pregnancy and previous studies have reported the inadequate iodine status of pregnant women in areas that have achieved iodine sufficiency in the general population. We examined the urinary iodine (UI) concentrations of pregnant women in Ukraine, where the iodine status is showing improvement among the general population.

Methods: We enrolled 148 pregnant women less than 16 weeks pregnant and 80 healthy women as a control group living in Zhitomir, Ukraine. UI concentration, thyroid-stimulating hormone (TSH), antithyroglobulin antibodies (TGAb), and antithyroid peroxidase antibodies (TPOAb) were measured.

Results: The median UI concentrations were significantly lower in pregnant women than in control women [13.0 (ND-51.0) $\mu \mathrm{g} / \mathrm{L}$ vs. 62.0 (35.3-108.5) $\mu \mathrm{g} / \mathrm{L}, \mathrm{p}<0.001]$. TSH concentrations were significantly lower in pregnant women than in control women [1.7 (1.2-2.7) IU/L vs. 2.2 (1.4-3.1) IU/L, p=0.011], but this difference disappeared when adjusted for age (2.1 $\pm 0.1 \mathrm{IU} / \mathrm{L}$ vs. $2.4 \pm 0.2 \mathrm{IU} / \mathrm{L}, \mathrm{p}=0.097)$. The frequency of TSH over 6.2 IU/L and the frequency of positive TGAb and/or TPOAb were not statistically different between groups ( $\mathrm{p}=0.70$ and $\mathrm{p}=0.48$, respectively). The UI concentrations of 
142 pregnant women (95.9\%) were less than $150 \mu \mathrm{g} / \mathrm{L}$, indicating insufficient iodine intake.

Conclusions: The UI concentration of pregnant women in Ukraine revealed severe iodine deficiency. Regular monitoring and appropriate nutrition education are essential because iodine deficiency can be easily prevented by adequate iodine intake. The risk of iodine deprivation during pregnancy needs to be assessed locally over time, because it may occur in areas that are not globally recognized as being iodine-deficient.

Key words: urinary iodine concentration, iodine deficiency, pregnancy, Ukraine

\section{List of Abbreviations}

antithyroglobulin antibodies (TGAb), antithyroid peroxidase antibodies (TPOAb), enzyme-linked immunosorbent assay (ELISA), International Council for the Control of Iodine Deficiency Disorders (ICCIDD), not detected (ND), thyroid-stimulating hormone (TSH), United Nations Children's Fund (UNICEF), urinary iodine (UI), World Health Organization (WHO). 


\section{Introduction}

On a worldwide basis, iodine deficiency is the single most important preventable cause of brain damage, despite the fact that iodine deficiency disorders are among the easiest and least expensive of all nutrient disorders to prevent. Although significant progress has been achieved to eliminate iodine deficiency by universal salt iodization programs (1), salt must not only reach the entire affected population, but it also needs to be adequately iodized for particular groups that are most susceptible, such as pregnant women and young children.

Pregnant women are at a higher risk for iodine deficiency than the general population due to increased thyroid hormone production, increased renal iodine losses, and fetal iodine requirements in pregnancy (2). When iodine deficiency takes place during fetal development, endemic goiter, cretinism, growth retardation, intellectual impairments, neonatal hypothyroidism, increased pregnancy loss, and increased infant mortality may occur (3). Therefore, the recommended adequate urinary iodine (UI) concentration for pregnant women ranges between 150 and $249 \mu \mathrm{g} / \mathrm{L}$, which is greater than the recommended value for non-pregnant women (100-199 $\mu \mathrm{g} / \mathrm{L})(1)$.

During 1991 to 1996, Ashizawa et al. first examined the UI concentrations of 953 
children in Zhitomir, Ukraine, which is the same area where we conducted this study (4).

The median UI concentration was $39 \mu \mathrm{g} / \mathrm{L}$, suggesting moderate iodine deficiency status in this area. Tronko et al. then reported the median UI concentration of $41.7 \mu \mathrm{g} / \mathrm{L}$ for 1998-2000 (Cycle 1) and 47.5 $\mu \mathrm{g} / \mathrm{L}$ for 2001-2003 (Cycle 2) in Ukraine (5). In Cycle 1, the lowest UI concentrations were found in Zhitomir. However, the situation in Zhitomir improved in Cycle 2, when access to public health messages and to salt fortified with iodine and iodine-rich food was likely to have increased. According to the WHO global database on iodine deficiency in 2002, the median UI concentration was $89.7 \mu \mathrm{g} / \mathrm{L}$ for women 15 to 49 years of age in Ukraine (6). In addition, we screened UI concentrations in a population residing in the Kiev region of Ukraine in 2005. In these subjects, the median UI concentration was $109 \mu \mathrm{g} / \mathrm{L}$ and only 10 subjects were in the concentration range between 20 and $49 \mu \mathrm{g} / \mathrm{L}$, with none below $20 \mu \mathrm{g} / \mathrm{L}$ (7).

The above results suggest that the iodine status in Ukraine might be improving, probably due to supplementation with iodized salt. However, there are no reports that have evaluated the iodine status in pregnant women, who are one of the most susceptible population groups. Therefore, in the present study, we investigated the UI concentrations of pregnant women in Ukraine, where the iodine status is showing improvement among the general population. 


\section{Subjects and Methods}

\section{Participants}

The study protocol was approved by the institutional review board of Korosten Inter-Area Medical Diagnostic Center, Korosten, Zhitomir, Ukraine. Informed consent was obtained from all participants. The subjects were 148 healthy pregnant women 17 to 40 years of age who were less than 16 weeks pregnant and who visited Korosten Inter-Area Medical Diagnostic Center for periodic prenatal examinations. As a control group, 80 healthy non-pregnant women 19 to 44 years of age were randomly selected from the general population who visited the same center for general health examinations. The exclusion criteria for pregnant women and the control group included a history of thyroid disease, renal insufficiency, and having delivered previous children with cretinism. None of the study participants received medication containing iodine.

\section{Laboratory Analyses}

Blood samples were collected for measurement of thyroid-stimulating hormone (TSH), antithyroglobulin antibodies (TGAb), and antithyroid peroxidase antibodies (TPOAb). Serum TSH, TGAb, and TPOAb levels were measured using a Stat Fax ${ }^{\circledR}$ model 303+ 
enzyme-linked immunosorbent assay (ELISA) (Awareness Technology, Inc., Palm City, FL, USA). Inter-assay coefficients of variation were $5.3 \%$ to $9.3 \%$ for TSH, $3.9 \%$ to 8.5\% for TGAb, and $1.5 \%$ to $2.9 \%$ for TPOAb. The laboratory reference range for TSH was $0.3-6.2 \mathrm{IU} / \mathrm{L}$. Values $<125 \mathrm{IU} / \mathrm{mL}$ for TGAb and $<20 \mathrm{IU} / \mathrm{ml}$ for TPOAb were considered negative.

We collected 2-mL spot urine samples to measure UI concentration. UI concentration was measured by a simple microplate method based on the Sandell-Kolthoff reaction (8), which incorporates both the reaction and digestion processes in a microplate format (9). Briefly, using a specially designed sealing cassette to prevent the loss of vapor and cross-contamination among plates, ammonium persulfate digestion was performed in a 96-well microtiter plate (MicroWell; Nalge Nunc International, Rochester, NY, USA) in an oven at $110^{\circ} \mathrm{C}$ for 60 minutes. After digestion, the mixture was transferred to a transparent microplate and the Sandell-Kolthoff reaction was performed at $25^{\circ} \mathrm{C}$ for 30 minutes. Finally, the UI concentration in each well was measured by a microplate reader at $405 \mathrm{~nm}$. The sensitivity of this method was $\geq 25 \mu \mathrm{g} / \mathrm{L}$. The intra-assay coefficient of variability was $5.5 \%$ at $44.2 \mu \mathrm{g} / \mathrm{L}, 3.1 \%$ at $164.0 \mu \mathrm{g} / \mathrm{L}$, and $1.3 \%$ at $414.9 \mu \mathrm{g} / \mathrm{L}$. The inter-assay coefficient of variability was $1.8 \%$ at $44.2 \mu \mathrm{g} / \mathrm{L}, 3.5 \%$ at $164.0 \mu \mathrm{g} / \mathrm{L}$, and $2.0 \%$ at $414.9 \mu \mathrm{g} / \mathrm{L}$. We considered the World Health Organization (WHO), United 
Nations Children's Fund (UNICEF), and the International Council for the Control of Iodine Deficiency Disorders (ICCIDD) -recommended epidemiological criteria based on median UI concentration for assessing iodine intake in pregnant women. According to this criteria, median UI concentration less than $150 \mu \mathrm{g} / \mathrm{L}$ define a population which has insufficient iodine intake, between 150 and $249 \mu \mathrm{g} / \mathrm{L}$ define as adequate iodine intake, between 250 and $499 \mu \mathrm{g} / \mathrm{L}$ define as above requirements, and more than 500 $\mu \mathrm{g} / \mathrm{L}$ define as excessive iodine intake (1).

\section{Statistical methods}

UI concentrations were expressed as medians $\left(25^{\text {th }}-75^{\text {th }}\right.$ percentiles $)$. Differences between the pregnant and control groups were evaluated using Mann-Whitney’s U-test for age, UI concentration, and TSH concentration. Frequencies of positive TGAb and TPOAb were evaluated by the $\chi^{2}$ test. TSH concentrations adjusted for age between the groups were compared by analysis of covariance. All statistical analyses were performed using SPSS Statistics $18.0^{\circledR}$ software for Windows (SPSS Japan, Tokyo, Japan). Probability values less than 0.05 were considered indicative of statistical significance. 


\section{Results}

Table 1 shows the characteristics of the study participants. Pregnant women were younger than the control women (pregnant women, 25.9 \pm 5.5 years; control women, 31.9 \pm 6.1 years; $\mathrm{p}<0.001$ ). The mean gestational age at examination was 12.2 weeks. UI concentrations were significantly lower in pregnant women than in control women [13.0 $\mu \mathrm{g} / \mathrm{L}(\mathrm{ND}-51.0 \mu \mathrm{g} / \mathrm{L})$ vs. $62.0 \mu \mathrm{g} / \mathrm{L}(35.3-108.5 \mu \mathrm{g} / \mathrm{L}), \mathrm{p}<0.001]$. TSH concentrations were significantly lower in pregnant women than in control women [1.7 IU/L (1.2-2.7 IU/L) vs. $2.2 \mathrm{IU} / \mathrm{L}(1.4-3.1 \mathrm{IU} / \mathrm{L}), \mathrm{p}=0.011)$, but this difference disappeared when adjusted for age (2.1 $\pm 0.1 \mathrm{IU} / \mathrm{L}$ vs. $2.4 \pm 0.2 \mathrm{IU} / \mathrm{L}, \mathrm{p}=0.097)$. Six pregnant women $(4.1 \%)$ showed low TSH concentrations less than $0.3 \mathrm{IU} / \mathrm{L}$, but none of the control women showed TSH concentrations less than 0.3 IU/L. Also, 4 pregnant women (2.7\%) and 3 control women (3.8\%) showed relatively high TSH concentrations more than 6.2 IU/L. The frequency of TSH more than 6.2 IU/L between the groups was not statistically different $(p=0.70)$. The frequency of positive TGAb was higher in the control group (8.8\%) than in the pregnant group $(2.7 \%)(\mathrm{p}=0.042)$. On the other hand, the frequency of TPOAb was not statistically different between the two groups $(8.8 \%$ in the control group and $8.1 \%$ in the pregnant group; $\mathrm{p}=0.87)$. The frequency of positive TGAb and/or 
TPOAb was also not statistically different $(12.6 \%$ in the control group and $9.5 \%$ in the pregnant group; $\mathrm{p}=0.48)$.

The number of cases with iodine deficiencies among pregnant and control women is shown in Table 2. Among 148 pregnant women, UI concentrations were less than 25 $\mu \mathrm{g} / \mathrm{L}$ in 74 women (50.0\%). Thirty-five pregnant women showed 25-49 $\mu \mathrm{g} / \mathrm{L}$ (23.7\%), 28 women showed 50-99 $\mu \mathrm{g} / \mathrm{L}(18.9 \%), 5$ women showed 100-149 $\mu \mathrm{g} / \mathrm{L}$ (3.4\%) and 6 women showed more than $150 \mu \mathrm{g} / \mathrm{L}$ (4.0\%). On the other hand, only 6 women showed less than $25 \mu \mathrm{g} / \mathrm{L}$ in the control group (7.5\%). Twenty-three control women showed 25-49 $\mu \mathrm{g} / \mathrm{L}$ (28.8\%), 30 women showed 50-99 $\mu \mathrm{g} / \mathrm{L}$ (37.5\%), 10 women showed 100-149 $\mu \mathrm{g} / \mathrm{L}$ (12.5\%), and 11 women showed more than $150 \mu \mathrm{g} / \mathrm{L}$ (13.8\%). The ratio was significantly different between the two groups $(\mathrm{p}<0.001)$. Figure 1 shows the distribution of UI concentrations between pregnant and control women. There were 142 pregnant women (95.9\%) whose iodine intake was insufficient and only 4 pregnant women (2.7\%) whose iodine intake was adequate based on the WHO/UNICEF/ICCIDD criteria for pregnant women. 


\section{Discussion}

In our cross-sectional study in Zhitomir, Ukraine, an iodine-deficient status was observed; UI concentrations were significantly lower in pregnant women than in control women $[13.0 \mu \mathrm{g} / \mathrm{L}(\mathrm{ND}-51.0 \mu \mathrm{g} / \mathrm{L})$ vs. $62.0 \mu \mathrm{g} / \mathrm{L}(35.3-108.5 \mu \mathrm{g} / \mathrm{L}), \mathrm{p}<0.001]$ and UI concentrations in 142 pregnant women (95.9\%) were less than $150 \mu \mathrm{g} / \mathrm{L}$, indicating insufficient iodine intake on the basis of WHO/UNICEF/ICCIDD criteria (1). The difference in UI concentrations between the pregnant and control women was obvious, even though there were no significant differences in the frequency of high TSH concentrations and the frequencies of TGAb and/or TPOAb. Also, there were no significant differences in TSH concentrations between the two groups after adjustment for age, regardless of progressive shifts toward higher concentrations with age (10). Low TSH concentration in pregnant women compared to control women is a natural consequence of the rise in human chorionic gonadotropin concentration in the end of the first trimester (11). Because low UI concentration in the first trimester of pregnancy is not reflected by high TSH concentration, we included pregnant women who were less than 16 weeks pregnant in this study, so the mean gestational age was 12.2 weeks. The median UI concentration of control women was $62.0 \mu \mathrm{g} / \mathrm{L}$ in this study, which was 
significantly lower compared to that in our previous study in the Kiev region (7). This discrepancy may be caused by the different frequencies of iodized salt intake between these studies’ participants.

Recently, several studies reported the inadequate iodine status of pregnant women in areas that have achieved iodine sufficiency in the general population. In an iodine-sufficient area in Bosnia and Herzegovina, the median UI concentration of 300 pregnant women was $142 \mu \mathrm{g} / \mathrm{L}$ (12). In Catalonia, Spain, where the government developed a public health action plan to eliminate iodine deficiency disorders, the median UI concentration among 600 pregnant women was $104 \mu \mathrm{g} / \mathrm{L}$ and $72 \%$ of these pregnant women had UI concentrations below $150 \mu \mathrm{g} / \mathrm{L}$ (13). Surprisingly, only $11 \%$ of these women reported using iodized salt in the food frequency questionnaire. A cross-sectional study in Lyon, France also suggested that pregnant women had an iodine-deficient status (14). Their median UI concentration was $81 \mu \mathrm{g} / \mathrm{L}$ and $77 \%$ had a UI concentration below $150 \mu \mathrm{g} / \mathrm{L}$. Mian et al. conducted screening in pregnant women from different geographic origins (Italy, Eastern Europe, Northern and Central Africa) and showed an overall median UI concentration of $83 \mu \mathrm{g} / \mathrm{L}$; the UI concentration was below $50 \mu \mathrm{g} / \mathrm{L}$ in 33\% and only 13\% had a UI concentration between 150 and $249 \mu \mathrm{g} / \mathrm{L}$, which is considered adequate according to the WHO/UNICEF/ICCIDD standards $(1,15)$. 
Our study in Ukraine is consistent with these previous studies, although the high frequency of low UI concentrations shows greater severity of iodine deficiency compared to the values of other countries.

Iodine requirement increases during pregnancy because of the increased requirement for thyroxine to maintain normal metabolism, the transfer of thyroxine and iodide from the mother to the fetus, and the increased loss of iodine in the urine resulting from an increased renal clearance of iodine. Beginning in early gestation, maternal thyroid hormone production normally increases by approximately 50\% in response to increased levels of serum thyroxine-binding globulin resulting from the increase in estrogen levels and stimulation of thyrotropin receptors by human chorionic gonadotropin. In addition, there is an increase in renal blood flow and glomerular filtration, which leads to an increased iodide clearance from the plasma and thus to an obligatory loss of iodine (2). Because the fetal thyroid does not begin to concentrate iodine until 10 to 12 weeks of gestation and is not controlled by fetal pituitary TSH until approximately 20 weeks of gestation (16), the fetus is reliant on the maternal thyroxine that crosses the placenta in very small quantities (17).

The WHO/UNICEF/ICCIDD guidelines recommend a daily iodine intake of 150 $\mu \mathrm{g} /$ day for non-pregnant adults and adolescents and $250 \mu \mathrm{g} /$ day for pregnant women (1). 
Increased hormone requirements can only be met by proportional increased hormone production, directly depending upon the availability of iodine in the diet. When dietary iodine is lacking, adequate physiology adaptation is difficult to achieve and is progressively replaced by pathological alterations occurring in parallel with the degree and duration of iodine deprivation, which leads to enhanced chronic glandular stimulation, hypothyroxinemia, and goiter formation (11).

Fish are known to contain relatively high amounts of iodine, as does iodized salt, an important dietary source of iodine provided in most populations as an intervention strategy for sustainable elimination of iodine deficiency and prevention $(1,18)$. However, changes in the diet during pregnancy are also necessary. Pregnant women are often advised to limit their salt intake to reduce swelling; therefore, implementation of strategies such as iodized salt may not always be feasible for pregnant women. It may be prudent to ensure adequate iodine intake during pregnancy by iodine supplementation, which is useful, safe, and beneficial for both the mother and the fetus during pregnancy. The American Thyroid Association recommends that women receive $150 \mu \mathrm{g} / \mathrm{L}$ iodine supplements daily during pregnancy and lactation. This is based on the fact that even though the median UI concentration of pregnant women was $173 \mu \mathrm{g} / \mathrm{L}$ (95\%CI: 75-229 $\mu \mathrm{g} / \mathrm{L}$ ), the lower 95\%CI was below $150 \mu \mathrm{g} / \mathrm{L}$ in the United States, 
which is recognized as an iodine-sufficient country (19).

One of the strengths of our study is that we conducted our research mainly during the first trimester of gestation. Because the main changes in thyroid function associated with pregnancy begin in the first trimester, sufficient iodine intake is indispensable before becoming pregnant as well. In an iodine-sufficient area, healthy women maintain iodine stores in the thyroid and during pregnancy; to help meet the increased iodine requirements, pregnant women draw on this iodine store. However, in areas of chronic iodine deficiency, women enter pregnancy with already depleted iodine stores (20). Our study showed that most of the pregnant women were iodine-depleted in the first trimester; therefore, we suggest that adequate iodine intake is important for not only pregnant women, but for all women of child-bearing age.

There are some limitations in this study. One is that we did not collect any dietary data to determine whether the study participants took any iodized preparations. In addition, we did not measure thyroid hormones, such as thyroxine and triiodothyronine. Another limitation is the relatively small sample size. Regardless of the iodine intake of a population, UI concentration varies considerably and the reliability of iodine nutrition studies and the number of samples necessary have not been settled. On the other hand, Andersen et al. suggested that the required numbers of urine samples needed to estimate 
the iodine intake level in a given population were 86 with a $90 \%$ confidence and $90 \%$ precision and 122 with a $95 \%$ confidence within a precision range of $\pm 10 \%$ (21). According to these standards, our number of samples appears to be adequate to assess the iodine status for this region.

In conclusion, the UI concentration of pregnant women in Ukraine revealed severe iodine deficiency. Regular monitoring and appropriate nutrition education are essential because iodine deficiency can be easily prevented by adequate iodine intake. The risk of iodine deprivation during pregnancy needs to be assessed locally over time, because it may occur in areas that are not globally recognized as being iodine deficient (22). The elimination of iodine deficiency disorders is a critical development issue and continued attention on the iodine status of pregnant women is necessary. 


\section{Acknowledgments}

This study was supported by Grant-in-Aid from the Japan Society for the Promotion of

Science (No.19500600) and the Ministry of Education, Culture, Sports, Science and Technology of Japan through the Nagasaki University Global COE program.

\section{Authors' conflict of interest disclosure}

The authors have nothing to declare. 


\section{References}

1. World Health Organization, United Nations Children's Fund, International Council for the Control of Iodine Deficiency Disorders. Assessment of iodine deficiency disorders and monitoring their elimination. A guide for programme managers, third edition. WHO, Geneva, Switzerland, 2007.

2. Glinoer D. The importance of iodine nutrition during pregnancy. Public Health Nutr 2007;10:1542-6.

3. Boyages SC. Clinical review 49: Iodine deficiency disorders. J Clin Endocrinol Metab 1993;77:587-91.

4. Ashizawa K, Shibata Y, Yamashita S, Namba H, Hoshi M, Yokoyama N, et al. Prevalence of goiter and urinary iodine excretion levels in children around Chernobyl. J Clin Endocrinol Metab 1997;82:3430-3.

5. Tronko M, Kravchenko V, Fink D, Hatch M, Turchin V, McConnell R, et al. Iodine excretion in regions of Ukraine affected by the Chornobyl accident: Experience of the Ukrainian-American cohort study of thyroid cancer and other thyroid diseases. Thyroid 2005;15:1291-7.

6. World Health Organization. WHO global database on iodine deficiency. Last 
updated 2006-12-28. Available at:

http://who.int/vmnis/iodine/data/database/countries/ukr_idd.pdf. Accessed: 20 May 2012.

7. Takamura N, Bebeshko V, Aoyagi K, Yamashita S, Saito H. Ukraine urinary iodine levels; 20 years after the Chernobyl accident. Endocr J 2007;54:335.

8. Sandell EB, Kolthoff IM. Micro determination of iodine by catalytic method. Microchem Acta 1937;1:9-25.

9. Ohashi T, Yamaki M, Pandav CS, Karmarkar MG, Irie M. (2000) Simple microplate method for determination of urinary iodine. Clin Chem 2000;46:529-36.

10. Surks MI, Hollowell JG. Age-specific distribution of serum thyrotropin and antithyroid antibodies in the U.S. population: implications for the prevalence of subclinical hypothyroidism. J Clin Endocrinol Metab, 2007;92:4575-82.

11. Glinoer D. Pregnancy and iodine. Thyroid 2001;11:471-81.

12. Tahirović H, Toromanović A, Balić A, Grbić S, Gnat D. Iodine nutrition status of pregnant women in an iodine-sufficient area. Food Nutr Bull 2009;30:351-4.

13. Alvarez-Pedrerol M, Ribas-Fitó N, García-Esteban R, Rodriguez À, Soriano D, Guxens $\mathrm{M}$ et al. Iodine sources and iodine levels in pregnant women from an area without known iodine deficiency. Clin Endocrinol 2010;72:81-6. 
14. Raverot V, Bournaud C, Sassolas G, Orgiazzi J, Claustrat F, Gaucherand P, et al. Pregnant French women living in the Lyon area are iodine deficient and have elevated serum thyroglobulin concentrations. Thyroid 2012;22:522-8.

15. Mian C, Vitaliano P, Pozza D, Barollo S, Pitton M, Callegari G, et al. Iodine status in pregnancy: role of dietary habits and geographical origin. Clin Endocrinol 2009;70:776-80.

16. Brown RS. Developmental regulation of thyrotropin receptor gene expression in the fetal and newborn thyroid. Endocrinology 2004;145:4058-61.

17. de Escobar GM, Obregon MJ, del Rey FE. Maternal thyroid hormones early in pregnancy and fetal brain development. Best Pract Res Endocrinol Metab 2004;18: 225-48.

18. Haldimann M, Alt A, Blanc A, Blondeau K. Iodine content of food groups. J Food Comp Anal 2005;18:461-71.

19. The Public Health Committee of the American Thyroid Association. Iodine Supplementation for Pregnancy and Lactation - United States and Canada: Recommendations of the American Thyroid Association. Thyroid 2006;16:949-51.

20. Zimmermann MB. Iodine deficiency. Endocr Rev 2009;30:376-408.

21. Andersen S, Karmisholt J, Pedersen KM, Laurberg P. Reliability of studies of iodine 
intake and recommendations for number of samples in groups and in individuals. $\mathrm{Br}$ J Nutr 2008;99:813-8.

22. Andersson M, de Benoist B, Rogers L. Epidemiology of iodine deficiency: salt iodisation and iodine status. Best Pract Res Endocrinol Metab 2010;24:1-11. 
Table 1. Characteristics of the study participants.

\begin{tabular}{l|lll} 
& Pregnant & Control & p-value \\
& $(n=148)$ & $(n=80)$ & \\
\hline Age (years) & $25.9 \pm 5.5$ & $31.9 \pm 6.1$ & $\mathrm{p}<0.001$ \\
UI $(\mu \mathrm{g} / \mathrm{L})$ & $13.0(\mathrm{ND}-51.0)$ & $62.0(35.3-108.5)$ & $\mathrm{p}<0.001$ \\
TSH (IU/L) & $1.7(1.2-2.7)$ & $2.2(1.4-3.1)$ & $\mathrm{p}=0.011$ \\
TGAb, $n$ (\%) & $4(2.7 \%)$ & $7(8.8 \%)$ & $\mathrm{p}=0.042$ \\
TPOAb, $n$ (\%) & $12(8.1 \%)$ & $7(8.8 \%)$ & $\mathrm{p}=0.87$ \\
TGAb and/or TPOAb, $n(\%)$ & $14(9.5 \%)$ & $10(12.6 \%)$ & $\mathrm{p}=0.48$
\end{tabular}

Values are mean \pm standard deviation or median $\left(25^{\text {th }}-75^{\text {th }}\right.$ percentile).

Abbreviations: urinary iodine (UI), thyroid-stimulating hormone (TSH), antithyroglobulin antibodies (TGAb), thyroid peroxidase antibodies (TPOAb), not detected (ND). 
Table 2. Urinary iodine concentrations of pregnant and control women and number of cases with iodine deficiencies.

\begin{tabular}{|c|c|c|c|c|c|c|}
\hline \multicolumn{2}{|c|}{ Number of samples } & \multirow{2}{*}{$<25 \mu \mathrm{g} / \mathrm{L}$} & \multirow{2}{*}{$\begin{array}{l}\text { 25-49 } \\
\mu g / L\end{array}$} & \multirow{2}{*}{$\begin{array}{l}\text { 50-99 } \\
\mu g / L\end{array}$} & \multirow{2}{*}{$\begin{array}{c}100-149 \\
\mu g / L\end{array}$} & \multirow{2}{*}{$\geq 150 \mu \mathrm{g} / \mathrm{L}$} \\
\hline & & & & & & \\
\hline Pregnant & 148 & 74 & 35 & 28 & 5 & 6 \\
\hline$(\%)$ & & $(50.0 \%)$ & $(23.7 \%)$ & (18.9\%) & $(3.4 \%)$ & $(4.0 \%)$ \\
\hline Control & 80 & 6 & 23 & 30 & 10 & 11 \\
\hline$(\%)$ & & $(7.5 \%)$ & $(28.7 \%)$ & (37.5\%) & (12.5\%) & $(13.8 \%)$ \\
\hline
\end{tabular}




\section{Figure Legends}

Figure 1. Distribution of urinary iodine concentrations in pregnant and control women. 


\section{Distribution of urinary iodine concentrations in pregnant and control women}

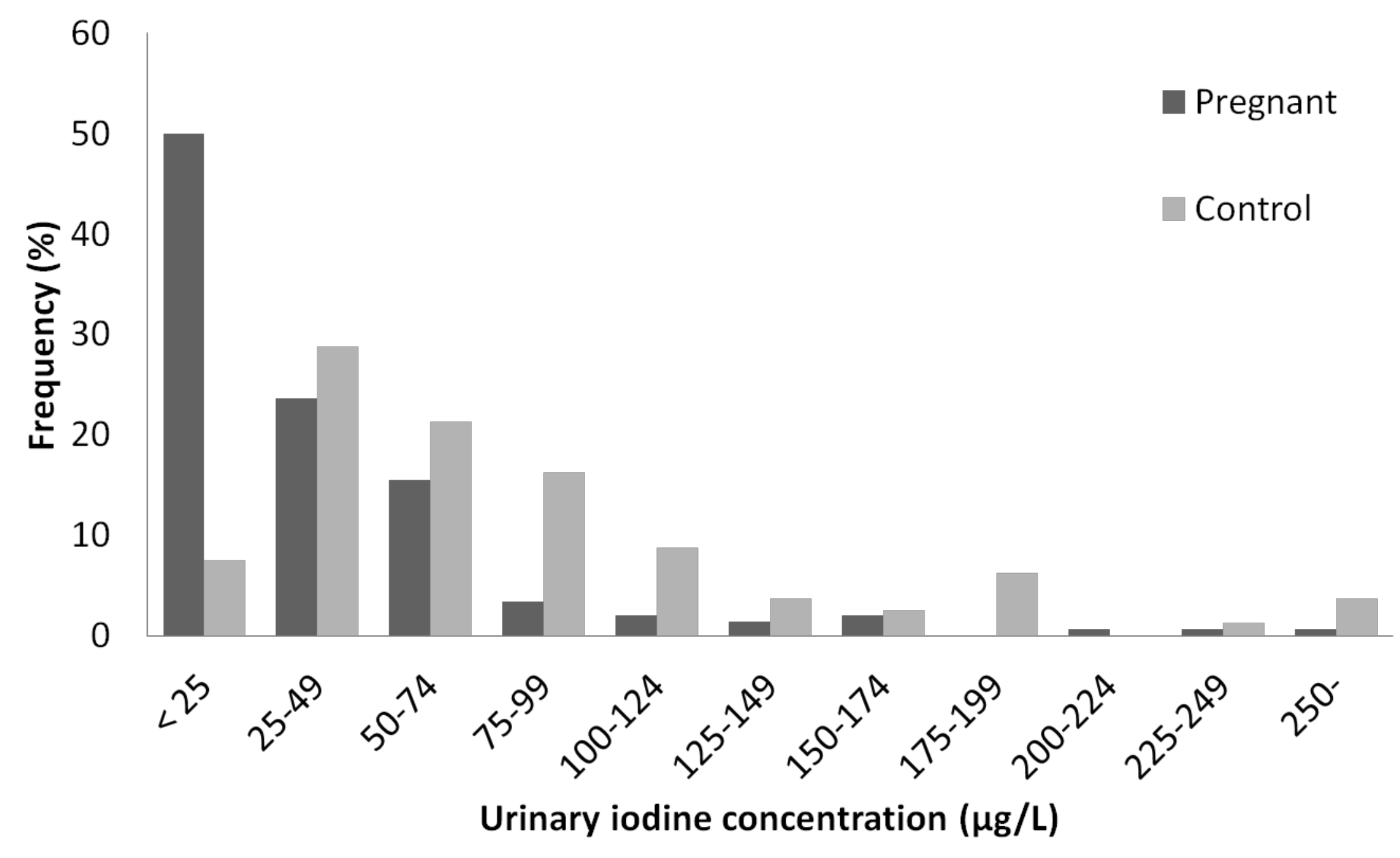

